\title{
Knowledge, Attitudes, Behaviors
}

National Cancer Institute

\section{Source}

National Cancer Institute. Knowledge, Attitudes, Behaviors. NCI Thesaurus. Code

C16772.

The consideration of knowledge, attitude, or behavior as variables in disease processes such as incident, transmission, and control. 